\title{
Fangchinoline exerts antitumour activity by suppressing the EGFR-PI3K/AKT signalling pathway in colon adenocarcinoma
}

\author{
FENGQI JIANG ${ }^{1,2}$, SHUO REN $^{1}$, YAODONG CHEN ${ }^{3}$, ANGE ZHANG $^{1}$,

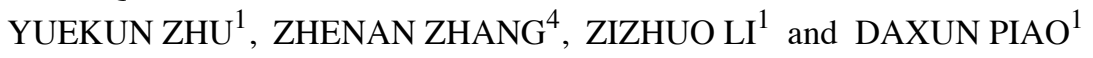 \\ ${ }^{1}$ Department of Colorectal Surgery, The First Affiliated Hospital of Harbin Medical University; \\ ${ }^{2}$ Department of General Surgery, Heilongjiang Provincial Hospital, Harbin, Heilongjiang 150001; \\ ${ }^{3}$ Department of Ultrasonic Imaging, First Clinical Medical College, Shanxi Medical University, \\ Taiyuan, Shanxi 030001; ${ }^{4}$ Department of Surgery, Infectious Disease Hospital of \\ Heilongjiang Province, Harbin, Heilongjiang 150001, P.R. China
}

Received February 27, 2020; Accepted October 7, 2020

DOI: $10.3892 /$ or.2020.7857

\begin{abstract}
Fangchinoline (FAN), an alkaloid extracted from Stephania tetrandra, has a variety of biological and pharmacological activities, but evidence of its effects on colon adenocarcinoma (COAD) is limited. Therefore, the present study aimed to elucidate the molecular mechanisms by which FAN affects COAD. The cytotoxicity, viability and proliferation of DLD-1 and LoVo cells were assessed in the presence of FAN using MTT and colony formation assays. The effects of FAN on apoptosis and the cell cycle in COAD cells were analysed by flow cytometry, and the migration and invasion of these cells were assessed by wound healing and Transwell experiments. Furthermore, a network pharmacological analysis was conducted to investigate the target of FAN and the results were confirmed by western blotting. In addition, a xenograft model was established in nude mice, and ultrasound imaging was used to assess the preclinical therapeutic effects of FAN in vivo. To the best of our knowledge, the results of this study provided the first evidence that FAN inhibited cellular proliferation, stemness, migration, invasion, angiogenesis and
\end{abstract}

Correspondence to: Dr Yuekun Zhu or Professor Daxun Piao, Department of Colorectal Surgery, The First Affiliated Hospital of Harbin Medical University, 23 Post Street, Harbin, Heilongjiang 150001, P.R. China

E-mail: zhuyuekun2011@126.com

E-mail: piaodaxun@sina.com

Abbreviations: FAN, fangchinoline; EGFR, epidermal growth factor receptor; CRC, colorectal cancer; COAD, colon adenocarcinoma; NPCs, natural plant compounds; EMT, epithelial-mesenchymal transition; CDFI, colour Doppler flow imaging; CPA, colour power angiography; USE, ultrasonic elastosonography; SRC, proto-oncogene tyrosine-protein kinase Src; ALB, albumin

Key words: FAN, colon adenocarcinoma, EGFR-PI3K/AKT signalling pathway, EMT epithelial-mesenchymal transition (EMT), and induced apoptosis and G1-phase cell cycle arrest. Network pharmacological analysis further confirmed that FAN prevented EMT through the epidermal growth factor receptor (EGFR)-phosphoinositide 3-kinase (PI3K)/AKT signalling pathway. Finally, FAN significantly repressed tumour growth and promoted apoptosis in xenografts. Thus, targeting EGFR with FAN may offer a novel therapeutic approach for COAD.

\section{Introduction}

Among all malignant tumours, colorectal cancer (CRC) ranks third in incidence and second in cancer-related mortality (1). There are $>1.8$ million new cases reported each year, accounting for $6.1 \%$ of all sites of cancers (2). In addition, the most common histopathological type of CRC is colon adenocarcinoma (COAD). Although significant advancements have been made in the early diagnosis and treatment of colon cancer, the long-term survival and prognosis of patients remain poor due to recurrence and metastasis (3). Drug resistance is the primary cause of cancer treatment failure and cancer-related deaths. Therefore, the research and development of novel antitumour drugs for metastasis has been a long-term focus of antitumour therapy. Plant-derived compounds have numerous advantages, including cost, stability, safety and multitargeting abilities, making them highly valued in clinical applications (4).

Fangchinoline (FAN), a bisbenzylisoquinoline alkaloid, is extracted from Stephania tetrandra, which exists widely in nature. FAN possesses a variety of biological and pharmacological activities, including anti-hypertensive and anti-atherosclerotic activity, enhanced immunity, platelet aggregation inhibition and histamine release (5). In previous years, researchers have demonstrated that FAN exhibits antitumor activity against a variety of malignant tumours, such as bladder cancer, prostate cancer, breast cancer, gastric cancer, hepatocellular carcinoma and melanoma (6), but its effects on CRC are unclear. Aggressive metastatic dissemination accounts for $\sim 90 \%$ of CRC-related deaths (7). The loss of cell polarity and a shift from an epithelial phenotype to 
a mesenchymal fibroblastic phenotype, a process known as epithelial-mesenchymal transition (EMT), promotes malignant cell metastasis through the extracellular matrix (ECM) to other tissues (8). Exogenous and endogenous cytokines work together to stimulate receptors on the cell surface and then activate signalling pathways to regulate EMT factors, eventually inducing EMT. For example, growth factors (including EGF, VEGF, TGF- $\beta$, Wnt, stromal cell-derived factor 1 and prostaglandin $\mathrm{E}_{2}$ ), cytokines (such as FAM3 metabolism regulating signalling molecule $\mathrm{C}$ and interleukins) and receptor tyrosine kinases [including epidermal growth factor receptor (EGFR), hepatocyte growth factor receptor and insulin-like growth factor 1 receptor] have been implicated in the regulation of EMT (9). Tyrosine kinase inhibitors and monoclonal antibodies against the extracellular domain of EGFR are now widely used for metastatic CRC treatment after failure of first-line chemotherapy regimens $(10,11)$. Therefore, targeting EGFR is important for treating CRC.

The aim of the present study was to evaluate the anticancer activities of FAN in COAD and the potential molecular mechanisms involved in vitro and in vivo. The present study confirmed that FAN, as an alkaloid chemotherapeutic drug, had antitumour activity in COAD cells.

\section{Materials and methods}

Cells and reagents. The COAD cell lines DLD-1 and LoVo and the human intestinal epithelial cell line NCM460 were purchased from FuHeng BioLogy Cell Centre. DLD-1 and NCM460 cells were cultured in Dulbecco's modified Eagle's medium (DMEM; Thermo Fisher Scientific, Inc.), while LoVo cells were cultured in RPMI-1640 medium (Gibco; Thermo Fisher Scientific, Inc.). Both media were supplemented with $10 \%$ foetal bovine serum (FBS; Gibco; Thermo Fisher Scientific, Inc.) and $1 \%$ antibiotic $(100 \mathrm{U} / \mathrm{ml}$ penicillin and 100 mg/ml streptomycin; Sigma-Aldrich; Merck KGaA). All cells were cultured in a standard humidified incubator at $37^{\circ} \mathrm{C}$ under an atmosphere with $5 \% \mathrm{CO}_{2}$. FAN was purchased from Aladdin Industrial Corporation (cat. no. F110200), which was dissolved in dimethyl sulfoxide (DMSO) as a $65.78 \mathrm{mmol} / \mathrm{l}$ stock solution.

MTT assay. The MTT assay was performed to measure the cytotoxicity of FAN on DLD-1, LoVo and NCM460 cells. Cells were seeded into 96 -well plates $\left(5 \times 10^{3}\right.$ per/well), incubated overnight, and treated with varying concentrations of FAN $(0-9 \mu \mathrm{M})$ at $37^{\circ} \mathrm{C}$ for $48 \mathrm{~h}$. Subsequently, MTT solution ( $5 \mathrm{mg} / \mathrm{ml}, 20 \mu \mathrm{l} /$ well; Sigma-Aldrich; Merck KGaA) was added to each well, followed by DMSO (150 $\mu \mathrm{l} /$ well) to dissolve the formazan crystals. Then, the absorbance at $490 \mathrm{~nm}$ in each well was read on a microplate reader (ELx808, BioTek Instruments, Inc.). Three biologically independent experiments were performed.

Plate colony formation assay. COAD cells $\left(1 \times 10^{3}\right)$ were seeded in a $6-\mathrm{cm}$ culture dish, incubated overnight, and treated with varying concentrations of FAN $(0-7 \mu \mathrm{M})$ for $5 \mathrm{~h}$. Then, the supernatant was replaced with regular medium containing 10\% FBS. After 2 weeks, the cells were fixed with methanol $(\geq 99.5 \%)$ at room temperature for $15 \mathrm{~min}$ and stained with a $0.5 \%$ crystal violet solution at room temperature for $10 \mathrm{~min}$. Three biologically independent experiments were conducted.

Cell cycle assay. After DLD-1 and LoVo cells were treated with FAN $(5 \mu \mathrm{M})$ for $48 \mathrm{~h}$, they were harvested, fixed in $75 \%$ ethanol at $4^{\circ} \mathrm{C}$ overnight, treated with RNase and stained with propidium iodide (Beijing 4A Biotech Co., Ltd.). Analysis of the cell cycle distribution was performed using flow cytometry (FACSCalibur; BD Biosciences). ModFit $\mathrm{LT}^{\mathrm{TM}}$ version 5.0 software (Verity Software House, Inc.) was used for analysis. Three biologically independent experiments were conducted.

Apoptosis assay. DLD-1 and LoVo cells were seeded in 6-well plates $\left(2.5 \times 10^{5} \mathrm{per} /\right.$ well $)$ and allowed to adhere overnight. Then, FAN (0-7 $\mu \mathrm{M})$ was added, and the cells were incubated for $48 \mathrm{~h}$ before being harvested and stained with an Annexin V-FITC/PI Apoptosis Detection kit (cat. no. FXP018; Beijing 4A Biotech Co., Ltd.). FACSCalibur and FACSDiva ${ }^{\mathrm{TM}}$ 6.1.3 software (BD Biosciences) were used to analyse apoptosis (early and late apoptosis). Three biologically independent experiments were conducted.

Wound healing assay. Monolayers (90\% confluency) of DLD-1 and LoVo cells in serum-free medium were scratched with a sterile 200- $\mu$ l pipette tip and then treated with different doses of FAN $(0-2 \mu \mathrm{M})$ for $24 \mathrm{~h}$. The scratch was imaged under a light microscope (magnification, x100), and wound closure was assessed at different time points (0, 24 and $48 \mathrm{~h})$. The widths of the scratches were analysed with ImageJ v1.8.0 (National Institutes of Health), and three biologically independent experiments were conducted.

Transwell assay. Migration and invasion experiments were performed with Transwell chambers. DLD-1 and LoVo cells were pretreated with different doses of FAN $(0-2 \mu \mathrm{M})$ for $24 \mathrm{~h}$ before they were resuspended in FBS-free medium and seeded into the upper chambers of the inserts $\left(1 \times 10^{4}\right.$ cells $/ 200 \mu 1$ medium per well). Then, $500 \mu \mathrm{l}$ medium containing $10 \% \mathrm{FBS}$ was added to the lower chamber to serve as a chemoattractant. For invasion analysis, $50 \mu 1$ Matrigel (BD Biosciences) was added to the upper chamber to coat the polycarbonate membrane at room temperature for $6 \mathrm{~h}$ before the cells were seeded. Following incubation for $48 \mathrm{~h}$, the cells on the upper surface of the membrane were wiped away, and the migrated or invaded cells were fixed with methanol at room temperature for $15 \mathrm{~min}$ and stained with a $0.5 \%$ crystal violet solution at room temperature for $20 \mathrm{~min}$. Under a light microscope (magnification, x100), 5 randomly selected fields were quantitatively analysed by recording the mean number of cells. Three biological experiments were performed.

Western blot analysis. COAD cells were treated with different concentrations of FAN $(0-7 \mu \mathrm{M})$ for $48 \mathrm{~h}$, after which they were lysed with RIPA buffer (cat. no. P0013B; Beyotime Institute of Biotechnology) containing protease and phosphatase inhibitors. After the lysates were centrifuged at $4^{\circ} \mathrm{C}$ for $10 \mathrm{~min}$ at $12,000 \mathrm{x} \mathrm{g}$, the supernatants were transferred to new test tubes. A BCA kit (cat. no. P0012S; Beyotime Institute 
of Biotechnology) was used to measure protein concentration. Proteins ( $25 \mu \mathrm{g} / \mathrm{lane})$ were separated by SDS-PAGE on $10 \%$ gels (Beyotime Institute of Biotechnology), and then electrotransferred onto polyvinylidene difluoride membranes (EMD Millipore). After the membranes were blocked with $5 \%$ non-fat milk in TBS with $0.1 \%$ Tween-20 for $1 \mathrm{~h}$ at room temperature, they were incubated overnight at $4^{\circ} \mathrm{C}$ with primary antibodies targeting the following proteins (as appropriate): Phosphorylated (p)-AKT (Ser473; polyclonal, rabbit anti-human IgG; 1:1,000; cat. no. 4060S; Cell Signaling Technology, Inc.), EGFR (polyclonal, rabbit anti-human IgG; 1:1,000; cat. no. 18986-1-AP; ProteinTech Group, Inc.), E-cadherin (polyclonal, rabbit anti-human $\mathrm{IgG}$; 1:1,000; cat. no. 20874-1-AP; ProteinTech Group, Inc.), $\mathrm{N}$-cadherin (polyclonal, rabbit anti-human IgG; 1:1,000; cat. no. 22018-1-AP; ProteinTech Group, Inc.), vimentin (polyclonal, rabbit anti-human IgG; 1:1,000; cat. no. 10366-1-AP; ProteinTech Group, Inc.), MMP2 (polyclonal, rabbit anti-human IgG; 1:1,000; cat. no. 10373-2-AP; ProteinTech Group, Inc.), MMP9 (polyclonal, rabbit anti-human IgG; 1:1,000; cat. no. 10375-2-AP; ProteinTech Group, Inc.), CD133 (polyclonal, rabbit anti-human $\operatorname{IgG}$; 1:1,000; cat. no. 66666-1-Ig; ProteinTech Group, Inc.), cyclin D1 (polyclonal, rabbit anti-human IgG; 1:1,000; cat. no. 60186-1-Ig; ProteinTech Group, Inc.), Bax (polyclonal, rabbit anti-human IgG; 1:1,000; cat. no. 50599-2-Ig; ProteinTech Group, Inc.), Bcl-2 (polyclonal, rabbit anti-human IgG; 1:1,000; cat. no. 12789-1-AP; ProteinTech Group, Inc.), zinc finger E-box-binding homeobox 1 (ZEB1; polyclonal, rabbit anti-human IgG; 1:1,000; cat. no. 21544-1-AP; ProteinTech Group, Inc.), ZEB2 (polyclonal, rabbit anti-human IgG; 1:1,000; cat. no. 14026-1-AP; ProteinTech Group, Inc.), zinc finger protein SNAI1 (SNAIL; polyclonal, rabbit anti-human IgG; 1:1,000; cat. no. 13099-1-AP; ProteinTech Group, Inc.), zinc finger protein SNAI2 (SLUG; polyclonal, rabbit anti-human IgG; 1:1,000; cat. no. 12129-1-AP; ProteinTech Group, Inc.), AKT (polyclonal, rabbit anti-human IgG; 1:1,000; cat. no. 10176-2-AP; ProteinTech Group, Inc.) and $\beta$-actin (polyclonal, mouse anti-human IgG; 1:1,000; cat. no. TA-09; OriGene Technologies, Inc.). Then, the membranes were incubated with anti-rabbit (HRP-conjugated goat anti-rabbit IgG; 1:10,000; cat. no. ZB-2301; OriGene Technologies, Inc.) or anti-mouse (HRP-conjugated goat anti-mouse IgG; 1:10,000; cat. no. ZB-2305; OriGene Technologies, Inc.) secondary antibodies for $1 \mathrm{~h}$ at room temperature. Protein signals were visualized using an enhanced chemiluminescence substrate (Thermo Fisher Scientific, Inc.) on a Bio-Rad gel imaging system (Bio-Rad Laboratories, Inc.). Image Lab version 2.0.1 (Bio-Rad Laboratories, Inc.) was used for semi-quantification. Three biologically independent experiments were performed.

Bioinformatics prediction. GEPIA2 (http://gepia2.cancer-pku. $\mathrm{cn}$ /\#index) analysis included differential expression in tumours vs. normal tissues, patient survival and multiple gene comparisons (12). The Human Protein Atlas (https://www.proteinatlas. org/) was used to determine the EGFR protein levels in colon cancer and normal colon tissues with an EGFR antibody (CAB000035). The Venny online tool (http://bioinfogp. cnb.csic.es/tools/venny/) was used to obtain the intersection between two sets of data groups.
Chemical database collection of the properties of FAN. The PubChem website (https://pubchem.ncbi.nlm.nih.gov/) was used to search for FAN and download its three-dimensional (3D) structure as an SDF file, which was imported into the PharmMapper website (http://www.lilab-ecust. $\mathrm{cn} /$ pharmmapper/) for compound-target gene prediction, and compound-target gene information annotation was obtained and transformed into standard gene symbols.

Prediction of target genes of compounds in colonic neoplasms. The Comparative Toxicogenomics Database (CTD; http://ctdbase.org/) was searched using the key word 'Colonic Neoplasms' to obtain the pathogenic genes related to this disease. The overlapping genes for the compounds and diseases were obtained through the Venny online tool (http://bioinfogp.cnb.csic.es/tools/venny/).

Functional enrichment analysis. The genes were entered into The Database for Annotation, Visualization and Integrated Discovery (DAVID; https://david.ncifcrf.gov/) for Kyoto Encyclopaedia of Genes and Genomes (KEGG) pathway (http://www.genome.jp/kegg/pathway.html) and Gene Ontology (GO; http://www.geneontology.org) enrichment analyses. 'Homo sapiens' and false discovery rate $(\mathrm{FDR})<0.05$ were selected as the filter criteria. The ggplot2.R package (version 0.0.1; https://cran.r-project. org/web/packages/ggplot2/) was used for image visualization.

Protein-protein interaction (PPI) network construction. The PPI network of the target genes was constructed using the Search Tool for the Retrieval of Interacting Genes (STRING) online tool (https://string-db.org/). R version 3.6.0 software (https://www.r-project.org/) was used to connect the adjacent nodes in the PPI network.

Animal experiments. A total of 10 female BALB/C nude mice (5-6 weeks old; weight, $18 \mathrm{~g}$ ) were purchased from Beijing Vital River Laboratory Animal Technology Co., Ltd. and reared in a pathogen-free environment under a 12/12 h light/dark cycle at room temperature. The mice had free access to food and water. The protocol relating to all animal experiments was discussed and approved by the Institutional Animal Care and Use Committee of Harbin Medical University (Harbin, China). The health and behaviour of the mice were monitored every day (12). Xenograft tumours were established by subcutaneously injecting $0.1 \mathrm{ml}$ DLD-1 cells $\left(1 \times 10^{7}\right.$ cells $\left./ \mathrm{ml}\right)$ in culture medium into the flanks of the mice. When the xenograft tumours were established, the mice were randomly divided into the treatment group $(n=5)$ or control group $(n=5)$ and intraperitoneally injected with FAN $(0.1 \mathrm{ml}, 0.5 \mathrm{mg} / \mathrm{ml})$ or PBS, respectively, three times per week for 4 weeks. After the intraperitoneal injection of $1 \%$ pentobarbital sodium into mice $(50 \mathrm{mg} / \mathrm{kg})$, the tumours were monitored by ultrasound imaging (USI). Analysis of tumour growth, angiogenesis, microangiogenesis and tumour hardness was performed with Philips IU Elite ultrasound system (Philips Healthcare), which includes four USI modes, namely, B-ultrasound (B-mode), colour Doppler flow imaging (CDFI), colour power angiography (CPA) and ultrasonic elastosonography (USE). The mice were anesthetized with sodium pentobarbital $(100 \mathrm{mg} / \mathrm{kg})$, followed by 
cervical dislocation at the end of the experiment. The tumours, hearts, livers, spleens and lungs were collected for pathological examination, and the tumour volumes were calculated using the following formula: Volume = length $\mathrm{x}$ width ${ }^{2} / 2$.

Pathological examination via immunohistochemistry (IHC) haematoxylin and eosin $(H \& E)$ and TUNEL apoptosis detection. For IHC, formaldehyde-fixed (at room temperature for $48 \mathrm{~h}$ ) and paraffin-embedded tumour tissues were sliced into $5-\mu \mathrm{m}$ thick sections. The sections were deparaffinized using xylene, rehydrated in a graded series of ethanol, incubated with $3 \% \mathrm{H}_{2} \mathrm{O}_{2}$ for $30 \mathrm{~min}$, and blocked with $10 \%$ normal goat serum (Beijing Solarbio Science \& Technology Co., Ltd.) at room temperature for $1 \mathrm{~h}$. Sections were then immunostained with antibodies against Ki67 (1:200; cat. no. WL01384a; Wanleibio Co., Ltd.), EGFR (1:200; cat. no. WL02312; Wanleibio Co., Ltd.), p-Bcl-2 (1:100; cat. no. WL01556; Wanleibio Co., Ltd.), N-cadherin (1:100; cat. no. WL01047; Wanleibio Co., Ltd.) and cyclin D1 (1:200; cat. no. WL01435a; Wanleibio Co., Ltd.) at $4^{\circ} \mathrm{C}$ overnight. Tissues were then incubated with HRP-labeled goat anti-rabbit IgG (1:500; cat. no. 31460; Thermo Fisher Scientific, Inc.) at $37^{\circ} \mathrm{C}$ for $60 \mathrm{~min}$, then immersed in PBS for 5 min three times. The colour was developed with a 3,3'-Diaminobenzidine Substrate Kit (cat. no. DA1010; Beijing Solarbio Science \& Technology Co., Ltd.) at $4^{\circ} \mathrm{C}$ overnight, following which the sections were counterstained with haematoxylin at room temperature for $3 \mathrm{~min}$. Five visual fields on each slide were randomly selected and photographed under a light microscope (magnification, $\mathrm{x} 400$ ).

For H\&E staining, tissues were fixed and embedded in paraffin, sliced into 5- $\mu \mathrm{m}$ thick sections, stained with haematoxylin at room temperature for $5 \mathrm{~min}$, treated with $1 \%$ acid ethanol for $3 \mathrm{sec}$, washed with distilled water, stained with eosin solution at room temperature for $3 \mathrm{~min}$, dehydrated with graded alcohol and washed with xylene. After drying, the mounted slides were examined and five visual fields were randomly selected on each slide and imaged using a light microscope (magnification, x200).

Apoptosis in pathological tissues was detected using a TUNEL Apoptosis Detection Kit (Wanleibio Co., Ltd.), and stained with hematoxylin for $2 \mathrm{~min}$ at room temperature. A total of five visual fields were randomly selected on each slide and imaged using a light microscope (magnification, $\mathrm{x} 400$ ). Three biologically independent experiments were performed.

Statistical analysis. Statistical analysis and probit regression analysis were conducted using SPSS 22.0 software (IBM Corp.). Data are presented as the mean \pm standard deviation. Differences between the means of two groups were assessed using an unpaired Student's t-test (independent). Differences among the means of multiple groups were assessed using one-way ANOVA followed by a Bonferroni post hoc test. $\mathrm{P}<0.05$ was considered to indicate a statistically significant difference.

\section{Results}

FAN suppresses viability and proliferation of COAD cells. The chemical structural formula and 3D structure of FAN are shown in Fig. 1A and B, respectively. To evaluate cytotoxicity,
COAD cells were incubated with multiple concentrations of FAN (0-9 $\mu \mathrm{M})$ for $48 \mathrm{~h}$. The MTT assay results showed that FAN significantly inhibited COAD cell viability in a dose-dependent manner (Fig. 1C). The 50\% inhibitory concentration $\left(\mathrm{IC}_{50}\right)$ values for DLD-1 and LoVo cells at $48 \mathrm{~h}$, according to probit regression analysis, were 4.53 and $5.17 \mu \mathrm{M}$, respectively. Notably, FAN showed minimal cytotoxicity towards NCM460 cells (Fig. S1). The colony formation assay results indicated that the number and size of colonies decreased in a dose-dependent manner (Fig. 1D and E).

Disordered cell cycle regulation is the basic mechanism of infinite and rapid cancer cell proliferation (13). As shown in Fig. 1F and G, FAN induced cell cycle arrest in DLD-1 and LoVo cells in the G1 phase, with the percentage of cells in the G1 phase cells increasing from 42.84 to $57.68 \%$ and from 46.30 to $53.25 \%$, respectively. The western blot assay results showed that the expression of CD133 and cyclin D1 in cells treated with FAN decreased in a dose-dependent manner (Figs. 1J and S2).

FAN induces apoptosis in COAD cells. Dysregulation of the apoptotic process is closely associated with tumour progression and resistance to chemotherapies (14). Therefore, exploring drug-induced apoptosis is crucial for elucidating anticancer mechanisms. The results showed that FAN significantly induced apoptosis in a dose-dependent manner (Fig. 1H and I), and western blot analysis revealed decreased Bcl-2 expression and increased Bax expression in FAN-treated COAD cells (Figs. 1J and S2).

FAN impedes COAD cell mobility. Generally, chemotherapy can not only induce apoptosis, but also weaken mobility in tumour cells (15). Tumour cell mobility is essential for metastasis and is typically assessed by wound healing and Transwell assays. Lower concentrations of FAN were administered $(0-2 \mu \mathrm{M})$ to avoid effects related to decreased viability. As shown in Fig. 2A and B, FAN significantly reduced the wound healing rate of COAD cells in a dose-dependent manner compared with the untreated control group. Consistent with these observations, Transwell assay results demonstrated that the migration and invasion of FAN-treated COAD cells decreased in a dose-dependent manner (Fig. 2C and D).

FAN inhibits EMT in COAD cells. Western blot analysis results showed that E-cadherin expression increased with increasing FAN concentration, whereas vimentin and $\mathrm{N}$-cadherin expression decreased compared with the control group. Furthermore, MMP2 and MMP9 expression levels were decreased by FAN compared with the control cells (Fig. 3A and B). With increasing FAN concentrations, SNAIL and ZEB2 expression decreased in a dose-dependent manner compared with the control group, and the expression of SLUG in DLD-1 and LoVo cell lines was not inhibited following treatment with FAN. Inhibition of ZEB1 expression in DLD-1 cells by FAN required a concentration $>7 \mu \mathrm{M}$ (Fig. 3C and D). The public GEPIA database showed that SNAIL expression was higher in COAD tissues compared with the adjacent tissues, and the overall survival (OS) of patients with COAD was decreased in the SNAIL-overexpression group from the GEPIA2 database (Fig. S3). 

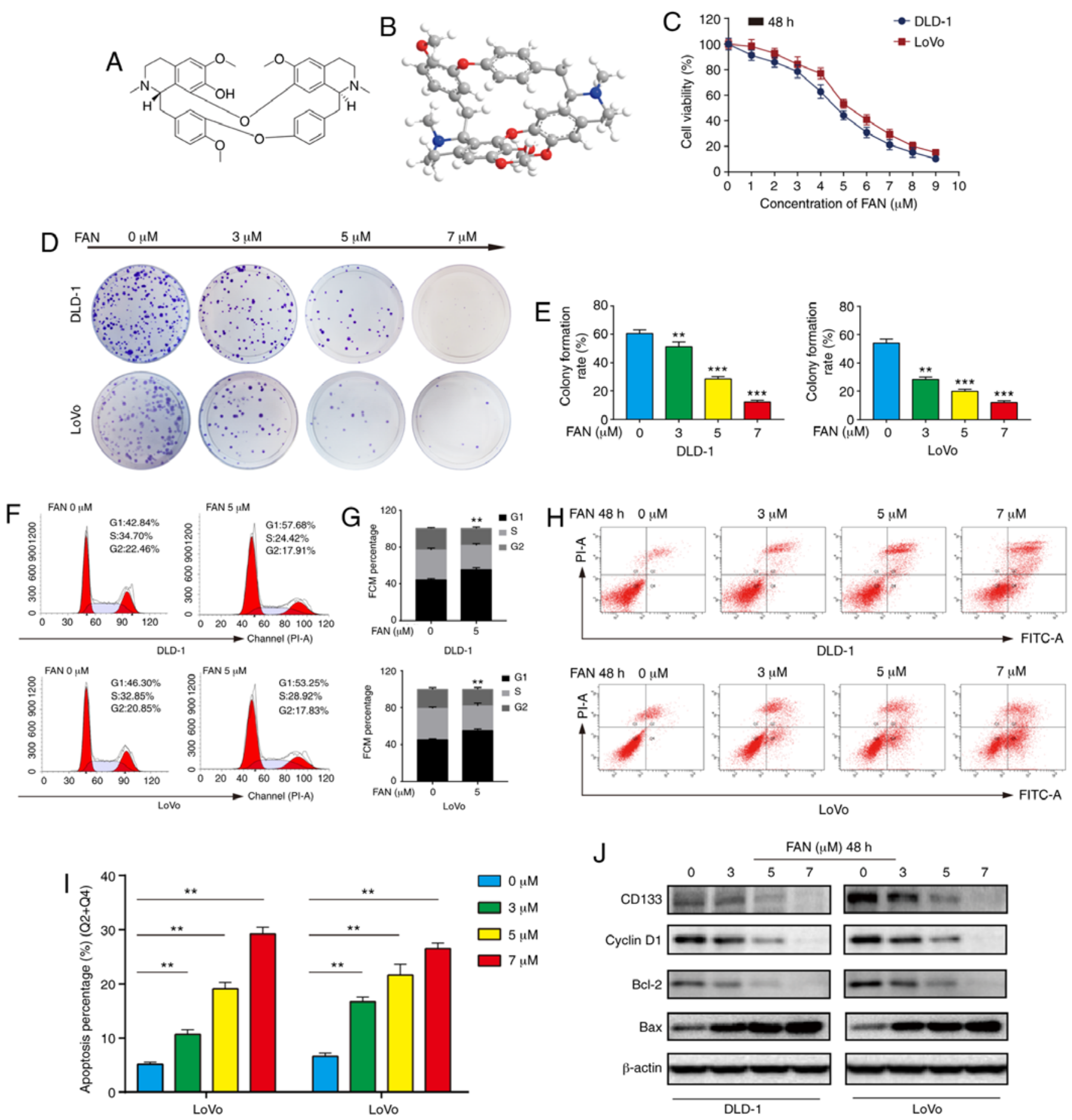

Figure 1. Effect of FAN on COAD cell proliferation, cell cycle and apoptosis. (A) Chemical and (B) three-dimensional structures of FAN. (C) An MTT assay was performed to measure COAD cell viability following FAN $(0-9 \mu \mathrm{M})$ treatment. (D and E) Colony formation assay of FAN ( $0-7 \mu \mathrm{M})$-treated COAD cells (F and G) Flow cytometry analysis of the cell cycle distribution of COAD cells after treatment with FAN $(5 \mu \mathrm{M})$. (H and I) Percentage of apoptotic COAD cells following treatment with FAN $(0-7 \mu \mathrm{M}, 48 \mathrm{~h})$ was determined by flow cytometry. (J) Western blot analysis of the expression of related proteins in COAD cells treated with FAN $(0-7 \mu \mathrm{M}) .{ }^{* *} \mathrm{P}<0.01$ and ${ }^{* * *} \mathrm{P}<0.001$ vs. $0 \mu \mathrm{M}$ group. FAN, fangchinoline; COAD, colon adenocarcinoma; FMC, flow cytometry.

FAN blocks the PI3K-AKT signalling pathway in COAD cells by inhibiting EGFR targets. The obtained SDF file for FAN, which was downloaded from the PubChem website, was imported into the PharmMapper website to predict target genes, and 258 compound-target genes were obtained. The CTD was searched with 'Colonic Neoplasms' as the key word and 25,581 pathogenic-target genes were obtained. Subsequently, 256 overlapping genes were identified as potential target genes through the online tool Venny (Fig. 4A).
A PPI network of five core potential target genes out of 256 potential target genes was constructed using the STRING online database (Fig. 4B). The circle in Fig. 4B represents the protein, and there are interactions between the linker proteins. $\mathrm{R}$ software was used to draw the number of adjacent nodes in the interactive network, where the abscissa is the number of adjacent nodes of the gene, and the ordinate is the name of the gene. Genes with more adjacent nodes are at the centre of the network. From the column (Fig. 4C), a number of potential 
A

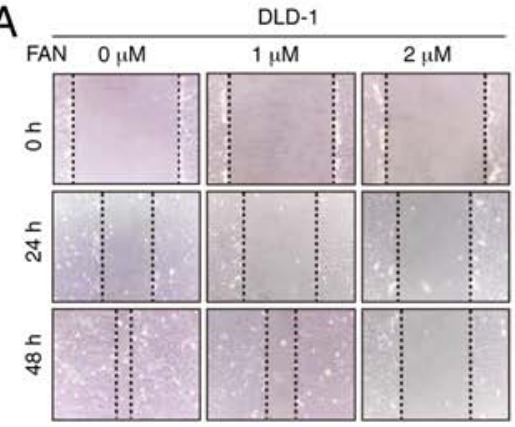

C

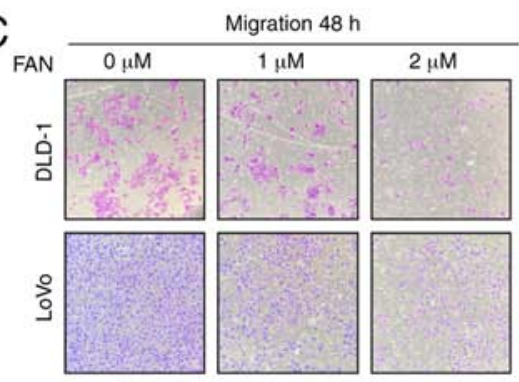

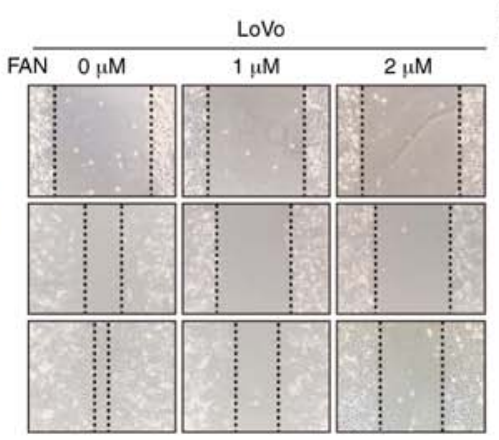

B
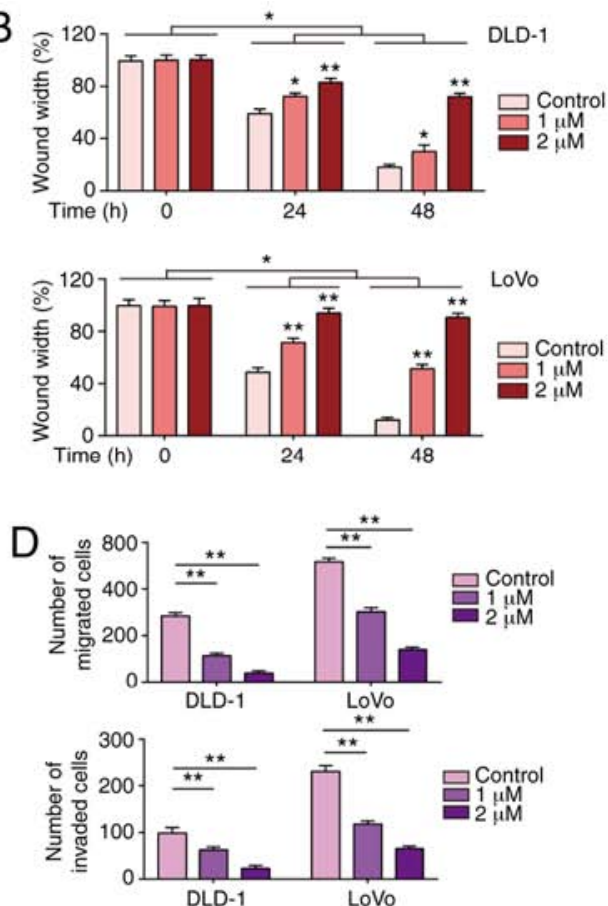

Figure 2. Effect of FAN on COAD cell mobility. (A and B) Wound healing assay of FAN-treated COAD cells at non-toxic concentrations $(0-2 \mu \mathrm{M}$, $24 \mathrm{~h}$ ). Mmagnification, $\mathrm{x} 100$. (C and D) Migration and invasion assays of COAD cells pretreated with FAN at non-toxic concentrations (0-2 $\mu \mathrm{M}, 24 \mathrm{~h})$. Mmagnification, $\mathrm{x} 100 .{ }^{*} \mathrm{P}<0.05$ and $^{* *} \mathrm{P}<0.01$. FAN, fangchinoline; COAD, colon adenocarcinoma.

A

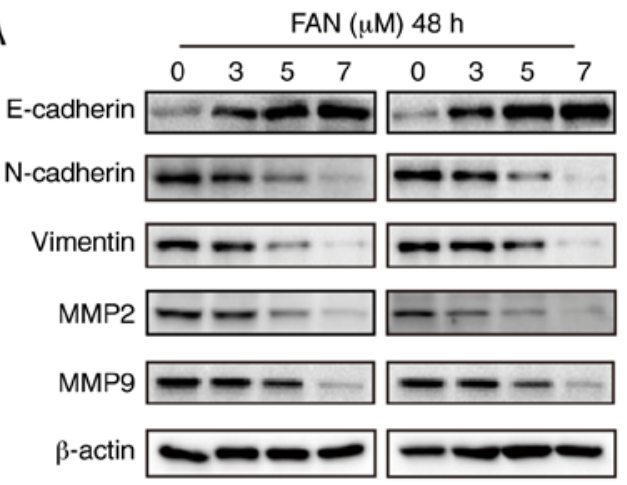

DLD-1
$B \div$

产

\section{든}

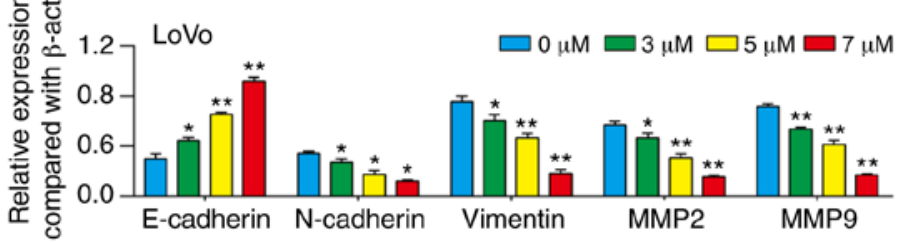

C

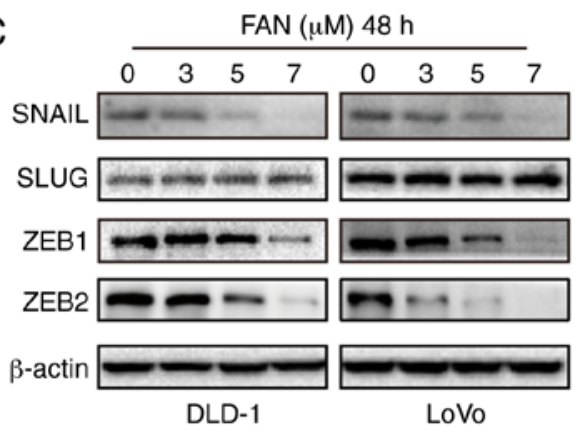

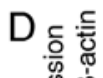
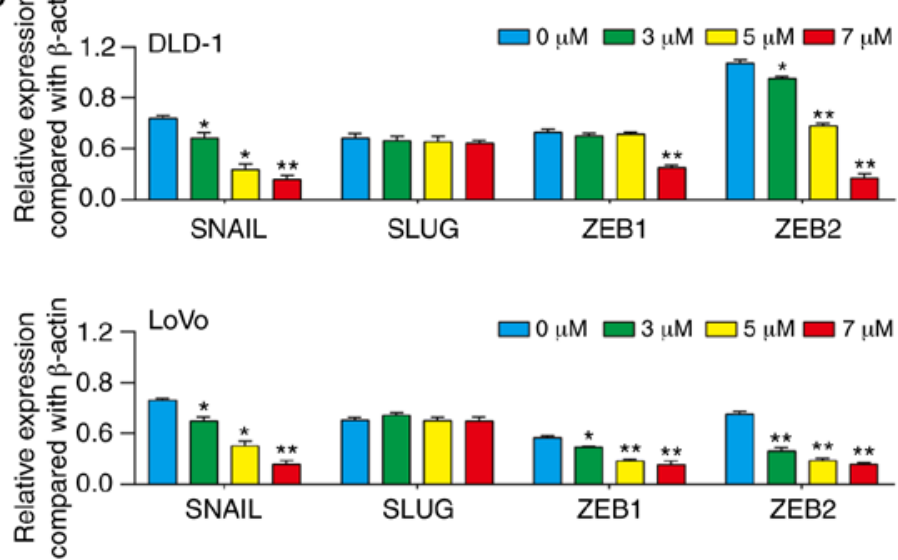

Figure 3. FAN represses EMT and EMT regulatory factors in colon adenocarcinoma cells. (A and B) E-Cadherin, N-cadherin, vimentin, MMP2 and MMP9 expression following treatment with FAN $(0-7 \mu \mathrm{M}, 48 \mathrm{~h})$ was determined by western blotting. (C and D) The expression of related transcription factors after FAN treatment $(0-7 \mu \mathrm{M}, 48 \mathrm{~h})$ was measured by western blotting. " $\mathrm{P}<0.05$ and ${ }^{* *} \mathrm{P}<0.01$ vs. $0 \mu \mathrm{M}$ group. FAN, fangchinoline; EMT, epithelial-mesenchymal transition; SNAIL, zinc finger protein SNAI1; SLUG, zinc finger protein SNAI2; ZEB1, zinc finger E-box-binding homeobox 1. 
A

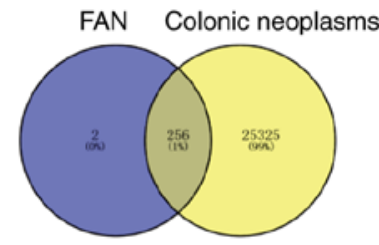

C

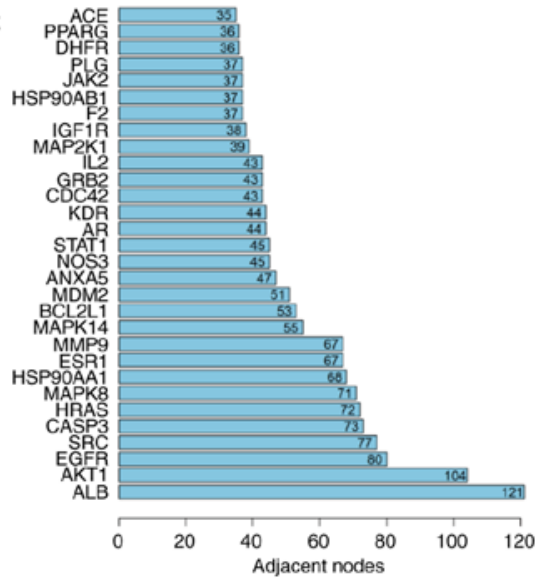

D

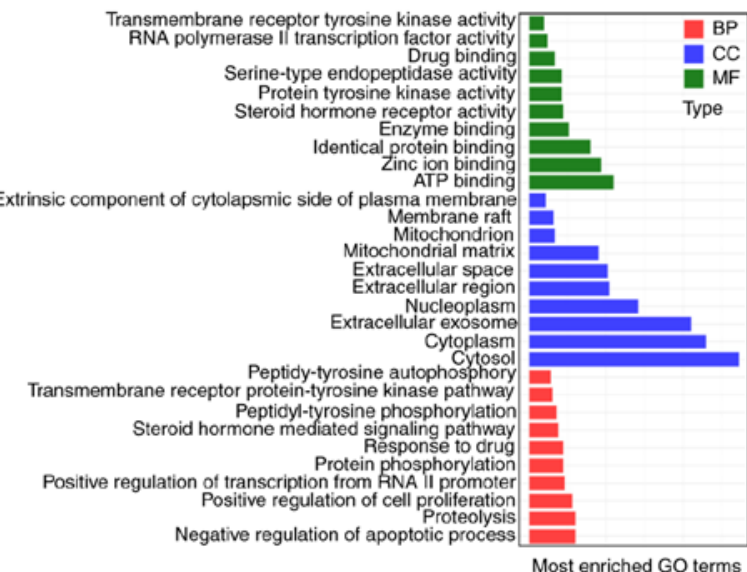

B

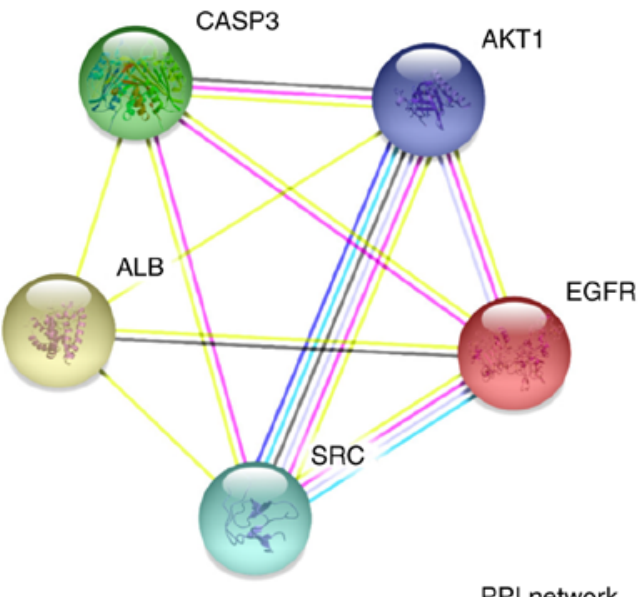

PPI network
E

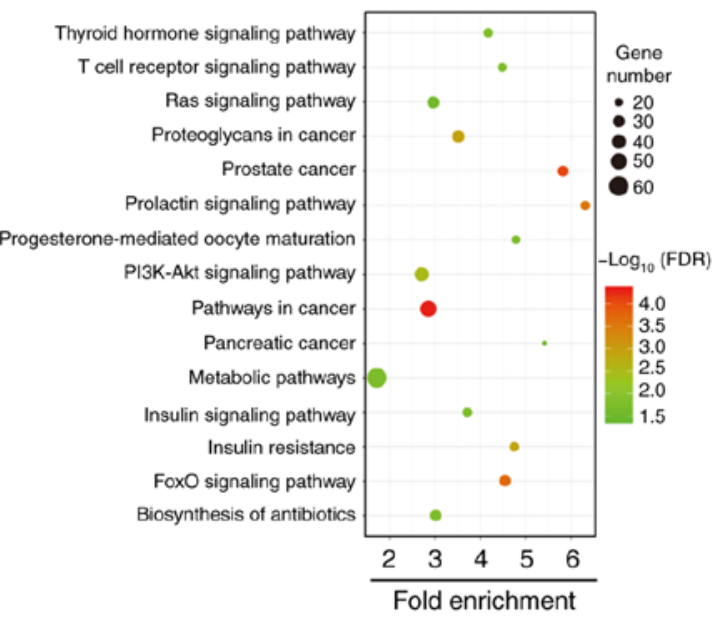

$\mathrm{F}$

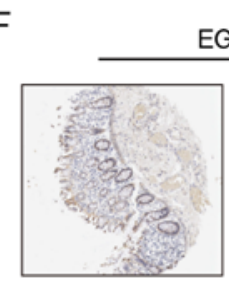

Normal
EGFR

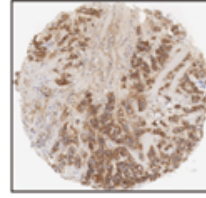

COAD
G
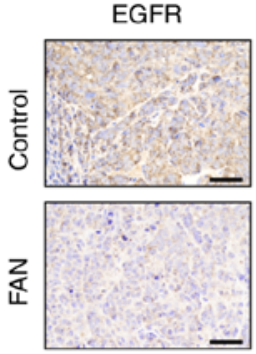

$\mathrm{H}$

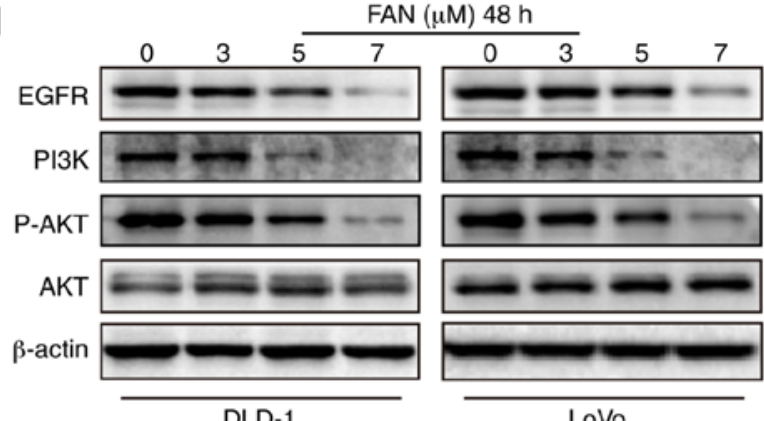

Figure 4. EGFR is a therapeutic target of colon cancer. (A) Venn diagram of the target genes for 258 compound-target genes and 25,581 pathogenic-target genes. (B) PPI network of the 5 core target genes among 256 potential target genes. (C) The column shows the adjacent nodes of the proteins. (D) GO analysis of potential target genes. (E) Kyoto Encyclopaedia of Genes and Genomes pathway analysis of potential target genes. (F) EGFR protein levels in normal and colon cancer tissues from The Human Protein Atlas database. (G) Immunohistochemistry analysis of EGFR expression in DLD-1 xenograft tumours following FAN treatment (magnification, $\mathrm{x} 400$; scale bar, $100 \mu \mathrm{m})$. (H) EGFR, PI3K, p-AKT and AKT expression levels in COAD cells treated with FAN $(0-7 \mu \mathrm{M}, 48 \mathrm{~h})$ were verified by western blotting. PPI, protein-protein interaction; EGFR, epidermal growth factor receptor; GO, Gene Ontology; FAN, fangchinoline; PI3K, phosphoinositide 3-kinase; p-, phosphorylated; ALB, albumin; SRC, proto-oncogene tyrosine-protein kinase Src; CASP3, caspase-3; BP, 'Biological Process'; MF, ‘Molecular Function'; CC, ‘Cellular Component'; COAD, colon adenocarcinoma.

target genes were obtained, among which albumin (ALB), AKT1, EGFR, proto-oncogene tyrosine-protein kinase Src (SRC) and caspase-3 (CASP3) were found to be at the core of the PPI network.
The 256 potential target genes were input into DAVID for GO enrichment analysis and KEGG signalling pathway enrichment analysis to elucidate the multiple biological functions of potential targets of FAN from a systematic 
A

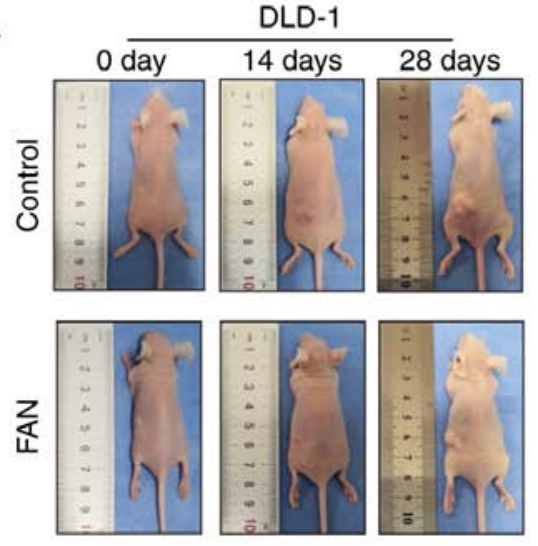

B
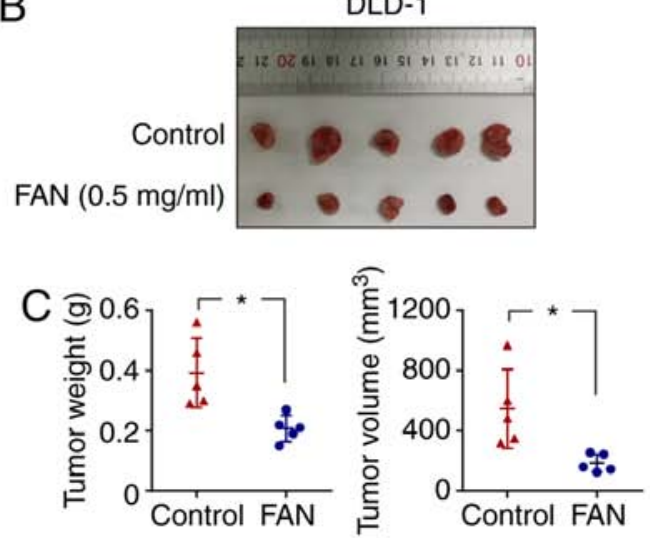

D
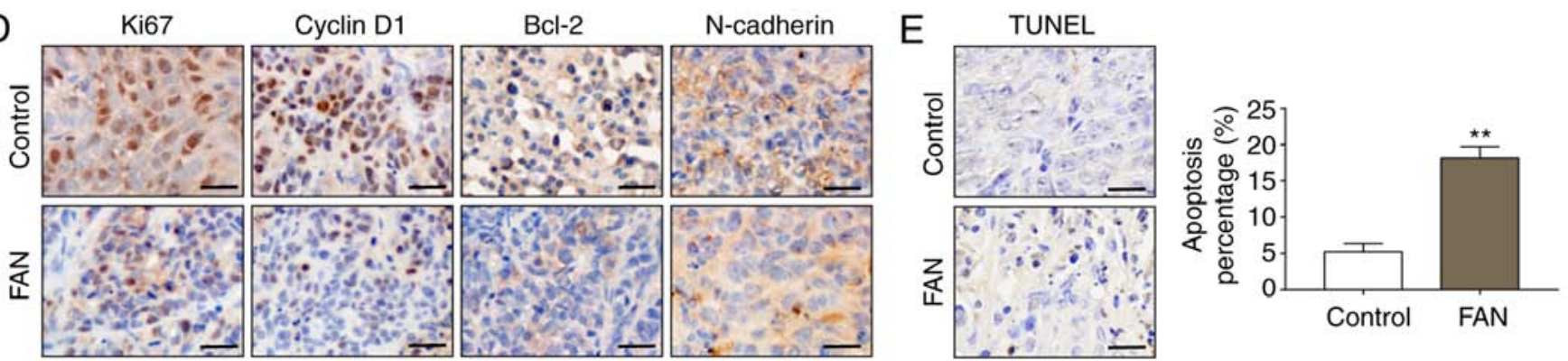

$\mathrm{F}$
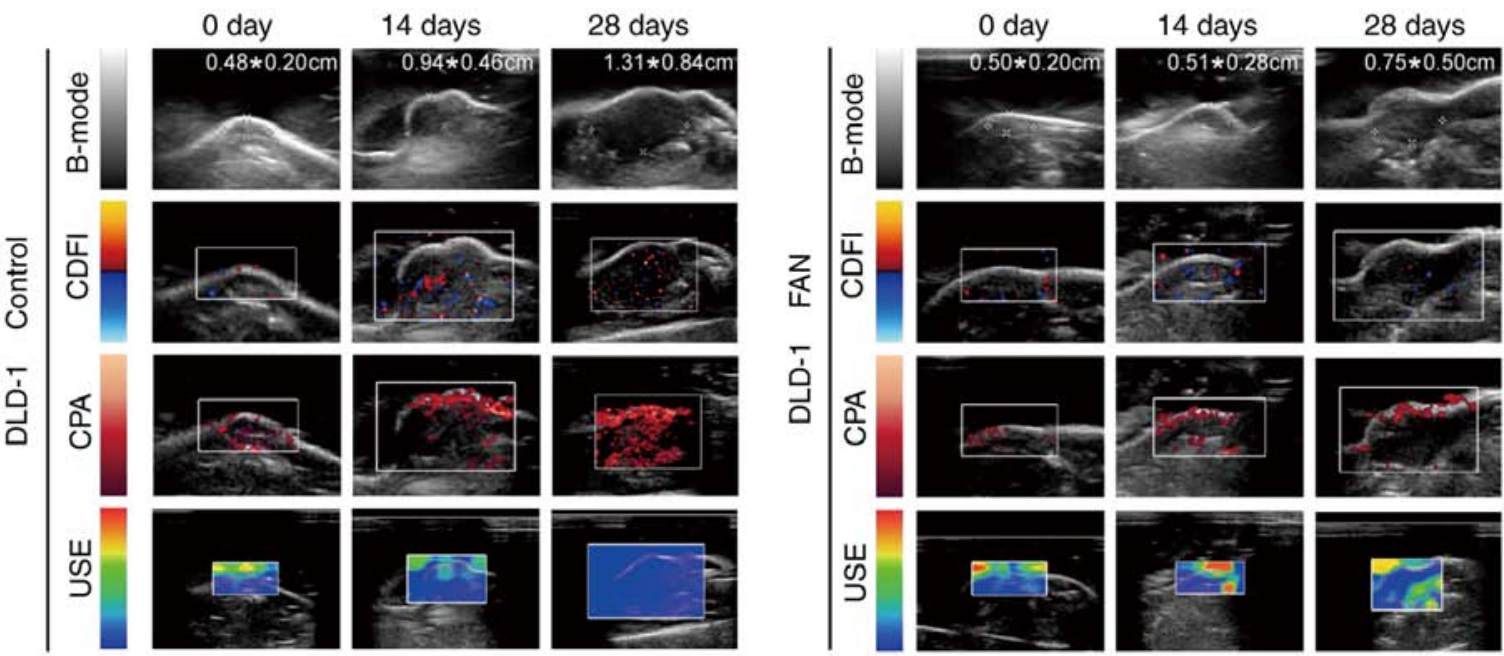

Figure 5. FAN suppresses tumour growth in vivo. (A-C) DLD-1 cells were subcutaneously injected into the left flanks of mice to establish xenograft models. Following FAN treatment $(0.1 \mathrm{ml}, 0.5 \mathrm{mg} / \mathrm{ml}$ three times per week for a total of 4 weeks), all mice were observed, and the tumour weight and volume were measured and compared. (D) Ki67, cyclin D1, Bcl-2 and N-cadherin expression levels in xenograft tumours were analysed by immunohistochemistry (magnification, $\mathrm{x} 400$; scale bar, $100 \mu \mathrm{m}$ ). (E) TUNEL assays were performed to detect apoptosis in pathological tissues. (F) Ultrasound evaluation that included B-mode, CDFI, CPA and USE. "P $<0.05$ and ${ }^{* * *} \mathrm{P}<0.01$ vs. control group. FAN, fangchinoline; B-mode, B-ultrasound; CDFI, colour Doppler flow imaging; CPA, colour power angiography; USE, ultrasonic elastosonography.

level (Fig. 4D and E). From the GO enrichment analysis for 'Biological Process', the potential targets of FAN were enriched in 'negative regulation of apoptotic process' (GO:0043066), 'proteolysis' (GO:0006508), 'positive regulation of cell proliferation' (GO:0008284), 'protein phosphorylation' (GO:0006468), 'response to drug' (GO:0042493) and others. For 'Cellular Component', the enriched items belonged to 'cytosol' (GO:0005829), 'cytoplasm' (GO:0005737), 'extracellular exosome' (GO:0070062), 'nucleoplasm' (GO:0005654), 'extracellular region' (GO:0005576) and others. For 'Molecular
Function', 'ATP binding' (GO:0005524), 'zinc ion binding' (GO:0008270), 'identical protein binding' (GO:0042802), 'enzyme binding' (GO:0019899) were primarily enriched (Fig. 4D). KEGG signalling pathway enrichment analysis results were enriched in 'Metabolic pathways' (hsa01100), 'Pathways in cancer' (hsa05200), 'PI3K-Akt signalling pathway' (hsa04151) and others (Fig. 4E).

A differential expression analysis of tumour vs. normal tissues showed that EGFR expression was higher in COAD tissues than in normal tissues (Fig. 4F). Furthermore, IHC 


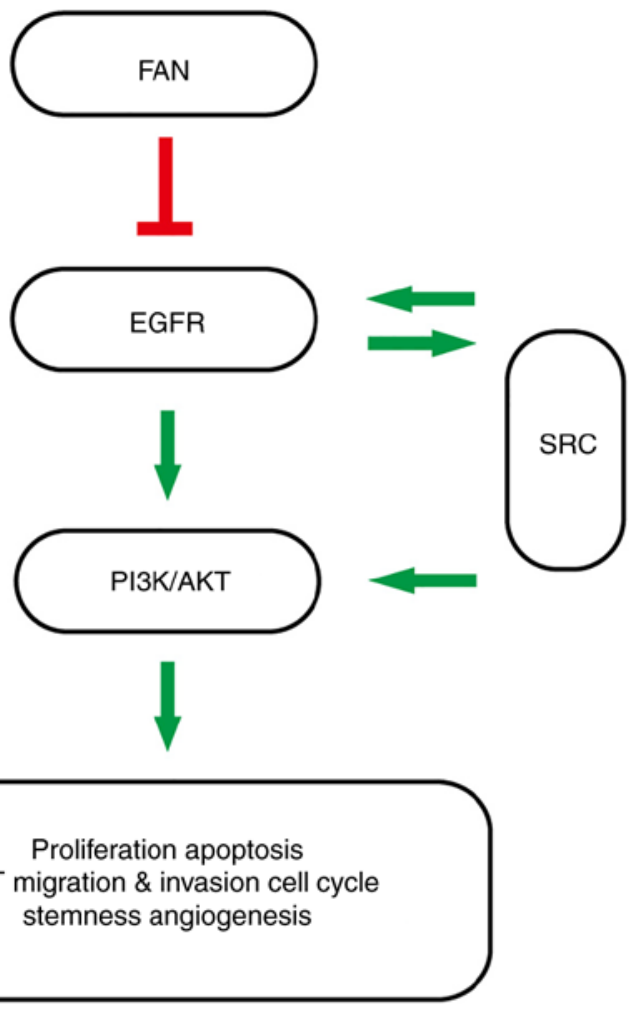

Figure 6. Molecular mechanism of FAN-induced colon cancer inhibition via the EGFR-SRC-PI3K/AKT signalling pathway. FAN, fangchinoline; EGFR, epidermal growth factor receptor; SRC, proto-oncogene tyrosine-protein kinase Src; PI3K, phosphoinositide 3-kinase; EMT, epithelial-mesenchymal transition.

results showed that EGFR expression was decreased in tumour tissues in the FAN treatment group (Fig. 4G). Moreover, the protein expression levels of EGFR, PI3K and p-AKT were decreased, but total AKT expression showed no significant changes (Figs. 4H and S4).

FAN suppresses tumour growth in vivo. USI (including B-mode, CDFI, CPA and USE) is a non-invasive imaging technique that can enable comprehensive analysis of xenograft tumours, and not only accurately measures the tumour size, but also displays the blood supply around the transplanted tumour and the hardness of the transplanted tumour. A DLD-1 xenograft model was established in BALB/c nude mice to evaluate the antitumour activity of FAN in vivo. After 4 weeks, the weights and sizes of the xenografts in the FAN treatment group were smaller than those observed in the control group, as shown in Fig. 5A-C. According to the different USI patterns in the FAN treatment group, the tumour size, as measured by the B-mode pattern, decreased, the xenograft tumour angiogenesis, as reflected by the CDFI and CPA patterns, decreased, and the xenograft tumour hardness, according to the USE pattern, decreased (Fig. 5F). Next, IHC was performed to measure the protein expression of representative tumour progression markers. Compared with that observed in the control group, the expression of Ki67, cyclin D1, Bcl-2 and N-cadherin in the FAN-treated group decreased (Fig. 5D). Haematoxylin and eosin (H\&E) staining of the FAN-treated group confirmed a reduction in tumour angiogenesis (Fig. S5B) without obvious necrosis in internal organs (Fig. S5A). The apoptotic rate of xenograft tumours, as determined by the TUNEL assay, was 17.23 and $3.05 \%$ in the FAN-treated and control groups, respectively, indicating the obvious apoptosis-inducing effect of FAN (Fig. 5E).

\section{Discussion}

Natural plant compounds (NPCs) have long been used as a source of therapeutic substances and a structural basis for drug development. Natural products provide unique structures that can be used to design novel therapeutic agents (16). Boldbaatar et al (17) systematically reviewed the anticancer, anti-inflammatory and antifibrosis effects of NPCs via EMT inhibition and concluded that NPCs inhibit pathological EMT through different signal transduction pathways in cells. In addition, accumulating evidence has suggested potential plant-derived compounds as inhibitors of several stages of tumorigenesis and of inflammatory and fibrotic processes (4). Several clinical trials have demonstrated that NPCs elicit anti-aging, anticancer and other health-enhancing effects. For example, berberine shows extensive pharmacological effects in gastroenteritis, abdominal pain and diarrhoea, and also antibacterial, anti-diabetic and anti-inflammatory properties (18). Furthermore, baicalin has been found to decrease blood lipids and inflammation in patients with coronary artery disease and rheumatoid arthritis, supporting its further clinical application (19). A low amount of gallic acid can prevent oxidative DNA injury and decrease inflammatory-, cancer- and cardiovascular disease-related markers (20). Key targets of the effects of NPCs may include the suppression of oxidative stress and the induction of 5'AMP-activated kinase (AMPK), or inhibition of the WNT/ $\beta$-catenin, PI3K/AKT/mTOR and RAS/MEK/ERK signalling pathways, which result in cell death and the prevention of conditions such as aging, diabetes, cardiovascular disease and cancer (21). During EMT, epithelial cells lose the expression of epithelial markers (including E-cadherin, occludin and cytokeratins) and begin to express mesenchymal markers (such as vimentin and fibronectin). In the present study, the expression of the epithelial marker E-cadherin increased significantly after FAN treatment, whereas that of the mesenchymal markers $\mathrm{N}$-cadherin and vimentin decreased significantly. Although it is unclear whether FAN can reverse EMT, it can be speculated that FAN may inhibit EMT. Cancer stem cells (CSCs) have the potential for self-renewal and multipotent differentiation, which leads to drug resistance (22). As a CSC surface marker, overexpression of CD133 is hypothesized to be involved in tumour metastasis and CRC relapse (23). Furthermore, degradation of the ECM due to increased MMP expression is crucial in the process of EMT-related metastasis (24,25). MMP2 and MMP9 are the most commonly used indicators of tumour invasion and metastasis. Western blot analysis in the present study showed that the expression levels of CD133, MMP2 and MMP9 were decreased significantly in the FAN-treated group. In addition, the expression of Bcl-2, an antiapoptotic marker, and cyclin D1, a cell cycle G1 marker, decreased with increasing FAN concentrations.

A group of transcription factors, namely, the EMT regulatory factors SNAIL, SLUG, ZEB1 and ZEB2, are upstream of the aforementioned markers and can regulate EMT by directly inhibiting E-cadherin promoter activity and promoting the 
mesenchymal phenotype (26-29). In the present study, the expression of SNAIL and ZEB2 decreased significantly and consistently in a dose-dependent manner in both COAD cell lines. A previous study demonstrated that the overexpression of SNAIL, a zinc finger transcriptional repressor, is closely associated with lymph node metastasis, indicating a poor clinical prognosis in patients with COAD, whereas blocking SNAIL protein expression can reduce the invasive activity of malignant tumours (30). ZEB2 expression has been demonstrated to be significantly higher in COAD tissues than in normal adjacent tissues (31), and Li et al (32) confirmed that ZEB2 promotes tumour metastasis and is associated with poor prognosis in patients with COAD. Therefore, we speculate that SNAIL and ZEB2 are primarily involved in regulating EMT as transcription factors in FAN-treated cells.

The concept of network pharmacology stems from the introduction of systems biology and the application of bioinformatics, as well as the 'genome' theory, which involves the integration of modern genomics, proteomics and metabolism (33). Based on the 'disease-gene-target-drug' interaction network, the intervention and influence of drugs on the disease network were analysed. The combination of a disease targeting database and molecular verification can provide a basis for identifying target genes and signalling pathways, as well as aiding the discovery of complex underlying mechanisms (34).

In the present study, through network pharmacological analysis, a PPI network of 256 potential target genes was constructed, and the molecular mechanisms were further predicted according to the enrichment of the potential target genes in the GO analysis and KEGG signalling pathways. The PPI network indicated that ALB, AKT1, EGFR, SRC and CASP3 were core components of tumour progression.

ALB, the most abundant protein in human blood, helps regulate plasma colloid osmotic pressure and acts as a carrier protein for a broad range of endogenous molecules (35). The loss of serum ALB reflects the degree of malnutrition and inflammatory response $(36,37)$. In a recent study, the combination of serum ALB and cholinesterase levels was revealed to be a useful clinical index, as it provided accurate prognostic information for patients with CRC (38). AKT1 is a member of the AKT family that plays a central role in the AKT pathway. A variety of biological processes, such as proliferation, cell cycle progression, invasion and metastasis, are regulated by AKT kinase (39). Caspase-3, a primary inducer of apoptosis, is activated directly by caspase-8, caspase- 9 and caspase-10, and exerts its function via convergence of the intrinsic and extrinsic apoptotic pathways $(40,41)$. SRC (a non-receptor tyrosine kinase) is a well-studied proto-oncogene that participates in the tumorigenesis of numerous types of cancer, such as colorectal, pancreatic cancer $(42,43)$. Studies have demonstrated that SRC cannot significantly increase the proliferation, invasiveness and tumorigenicity of tumour cells unless bound with EGFR and p-EGFR (42). As a component of EGFR signalling transduction, cellular SRC (C-SRC) mediates EGFR-driven tumorigenesis in vivo, and the interaction between EGFR and C-SRC leads to a more aggressive tumour phenotype $(44,45)$. Previously, a study on genetic variations in the EGFR pathway demonstrated that the interaction between
SRC and single nucleotide polymorphisms (SNPs) of EGFR is significantly related to the risk of COAD, which further supports the joint regulatory model of EGFR and SRC in early-stage colorectal cancer (46). Furthermore, SRC plays a central role in regulating multiple downstream pathways, such as the PI3K/AKT, signal transducer and activator of transcription 3 and mitogen-activated protein kinase pathways (47). SRC activation is associated with chemotherapy and EGFR antibody resistance, which has led to the strategy of developing SRC family-specific inhibitors to prevent immune inhibition (46). Research on NPCs targeting EGFR has remained important (48). The ligands of EGFR (EGF and TGF- $\alpha$ ) bind to its extracellular domain and induce phosphorylation of its intracellular domain, in turn activating an intricate downstream signalling pathway $(49,50)$ Curcumin treatment has been found to significantly reduce the proliferation and migration of NSCLC cells, possibly through toll-like receptor 4/myeloid differentiation primary response protein MyD88-EGFR-mediated inhibition of AP-1 protein and suppression of the EMT process (51). A study by Li et al (52) revealed that evodiamine can downregulate the expression of total EGFR in human LoVo cells, which may be the underlying mechanism of its inhibitory effects on invasion and metastasis of CRC via the PI3K, AKT and mTOR signalling pathways. The present study confirmed by western blotting that the expression of EGFR decreased in a dose-dependent manner. After a comprehensive analysis of the aforementioned potential targets, it was concluded that EGFR is the most likely target of FAN in the treatment of CRC.

To elucidate the molecular mechanism by which FAN affects COAD, GO analysis and KEGG pathway enrichment analysis of the 256 potential targets were performed. The KEGG pathway analysis results primarily identified the PI3K/AKT signalling pathway, which was consistent with previous reports on hepatocellular carcinoma and gastric cancer $(6,53)$. Abnormal activation of the PI3K/AKT signalling pathway leads to an imbalance between cell proliferation and apoptosis, resulting in tumorigenesis (54). In addition, terms in the 'Biological Process' category ranked highest among the top $10 \mathrm{GO}$ terms, suggesting that FAN may exert its anticancer activity by regulating different biological processes.

In a previous study, Wang et al (53) observed that FAN induced autophagy in HepG2 and PLC/PRF/5 cells via AMPK signalling. Feng et al (6) confirmed that FAN targets PI3K and suppresses the PI3K/AKT signalling pathway in SGC7901 cells. Shi et al (55) observed that FAN inhibits the growth and metastasis of melanoma cells by suppressing the focal adhesion kinase 1 pathway. These data indicate that FAN is a non-specific drug. Compared with single-target inhibitors, non-specific drugs have innate advantages, such as greater anticancer effects and a significantly reduced risk of multidrug resistance and serious side effects (56). Moreover, with the application of nanotherapy-based drug delivery systems, targeted and sustained drug release can be achieved, the solubility and bioavailability of drugs can be improved, and the side effects of drugs can be reduced (57). When combined with EGFR-targeted nanotherapy, plant-derived evodiamine showed a stronger ability to inhibit the invasion and metastasis 
of CRC than other monotherapy groups (51). Therefore, it is proposed that FAN could potentially have useful clinical applications in the future.

The present study confirmed that FAN has a strong inhibitory effect on tumorigenesis in vitro and in vivo. FAN can inhibit the proliferation and invasion of tumour cells and induce apoptosis and cell cycle arrest through the EGFR-PI3K/AKT signalling pathway (Fig. 6). In addition, SRC may be involved in the EGFR-SRC-PI3K/AKT signalling pathway according to the network pharmacology assessment and relevant literature reports; however, this finding needs further experimental confirmation. Therefore, these results suggested that FAN may be a potential anticancer strategy for COAD.

\section{Acknowledgements}

Not applicable.

\section{Funding}

The present work was supported by the National Natural Science Foundation of China (grant no. 81902468) and the Innovation Project of Harbin Medical University (grant no. YJSKYCX2018-37HYD).

\section{Availability of data and materials}

The datasets used and/or analysed during the current study are available from the corresponding author on reasonable request.

\section{Authors' contributions}

FJ, YZ and DP conceived and designed the present study. FJ and SR performed the experiments. FJ prepared and wrote the manuscript. ZL and YC conducted the ultrasonic imaging experiments. $\mathrm{ZZ}$ and $\mathrm{YZ}$ performed the pathology experiments. AZ was involved in the analysis and interpretation of data. YZ revised the manuscript for important intellectual content. All authors read and approved the final manuscript.

\section{Ethics approval and consent to participate}

All animal studies were approved by the Ethics Committee of The First Affiliated Hospital of Harbin Medical University (Harbin, China) and conducted in accordance with the national regulations of China.

\section{Patient consent for publication}

Not applicable.

\section{Competing interests}

The authors declare that they have no competing interests.

\section{References}

1. Banerjee A, Pathak S, Subramanium VD, G D, Murugesan R and Verma RS: Strategies for targeted drug delivery in treatment of colon cancer: Current trends and future perspectives. Drug Discov Today 22: 1224-1232, 2017.
2. Bray F, Ferlay J, Soerjomataram I, Siegel RL, Torre LA and Jemal A: Global cancer statistics 2018: GLOBOCAN estimates of incidence and mortality worldwide for 36 cancers in 185 countries. CA Cancer J Clin 68: 394-424, 2018.

3. Iqbal A and George TJ: Randomized clinical trials in colon and rectal cancer. Surg Oncol Clin N Am 26: 689-704, 2017.

4. Avila-Carrasco L, Majano P, Sánchez-Toméro JA, Selgas R, López-Cabrera M, Aguilera A and González Mateo G: Natural plants compounds as modulators of epithelial-to-mesenchymal transition. Front Pharmacol 10: 715, 2019.

5. Wang Y, Chen J, Wang L, Huang Y, Leng Y and Wang G: Fangchinoline induces $\mathrm{G} 0 / \mathrm{G} 1$ arrest by modulating the expression of CDKN1A and CCND2 in K562 human chronic myelogenous leukemia cells. Exp Ther Med 5: 1105-1112, 2013.

6. Feng T, Ding D and Li DD: Fangchinoline targets PI3K and suppresses PI3K/AKT signaling pathway in SGC7901 cells. Int J Oncol 46: 2355-2363, 2015.

7. Wu WK, Wang XJ, Cheng AS, Luo MX, Ng SS, To KF, Chan FK, Cho $\mathrm{CH}$, Sung JJ and Yu J: Dysregulation and crosstalk of cellular signaling pathways in colon carcinogenesis. Crit Rev Oncol Hematol 86: 251-277, 2013.

8. Lamouille S, Xu J and Derynck R: Molecular mechanisms of epithelial-mesenchymal transition. Nat Rev Mol Cell Biol 15: 178-196, 2014.

9. Barr S, Thomson S, Buck E, Russo S, Petti F, Sujka-Kwok I, Eyzaguirre A, Rosenfeld-Franklin M, Gibson NW, Miglarese M, et al: Bypassing cellular EGF receptor dependence through epithelial-to-mesenchymal-like transitions. Clin Exp Metastasis 25: 685-693, 2008.

10. Kim N, Cho D, Kim H, Kim S, Cha YJ, Greulich H, Bass A, Cho HS and Cho J: Colorectal adenocarcinoma-derived EGFR mutants are oncogenic and sensitive to EGFR-targeted monoclonal antibodies, cetuximab and panitumumab. Int J Cancer 146: 2194-2200, 2020.

11. Padfield E, Ellis HP and Kurian KM: Current therapeutic advances targeting EGFR and EGFRvIII in glioblastoma. Front Oncol 5: 5, 2015

12. Li Z, Chen Y, An T, Liu P, Zhu J, Yang H, Zhang W, Dong T, Jiang J, Zhang Y, et al: Nuciferine inhibits the progression of glioblastoma by suppressing the SOX2-AKT/STAT3-Slug signaling pathway. J Exp Clin Cancer Res 38: 139, 2019.

13. Vermeulen K, Berneman ZN and Van Bockstaele DR: Cell cycle and apoptosis. Cell Prolif 36: 165-175, 2003.

14. Russo A, Terrasi M, Agnese V, Santini D and Bazan V: Apoptosis: A relevant tool for anticancer therapy. Ann Oncol 17 (Suppl 7): vii115-vii123, 2006.

15. Qian L, Liu Y, Xu Y, Ji W, Wu Q, Liu Y, Gao Q and Su C: Matrine derivative WM130 inhibits hepatocellular carcinoma by suppressing EGFR/ERK/MMP-2 and PTEN/AKT signaling pathways. Cancer Lett 368: 126-134, 2015.

16. Sak K: Cytotoxicity of dietary flavonoids on different human cancer types. Pharmacogn Rev 8: 122-146, 2014.

17. Boldbaatar A, Lee S, Han S, Jeong AL, Ka HI, Buyanravjikh S, Lee JH, Lim JS, Lee MS and Yang Y: Eupatolide inhibits the TGF- $\beta 1$-induced migration of breast cancer cells via downregulation of SMAD3 phosphorylation and transcriptional repression of ALK5. Oncol Lett 14: 6031-6039, 2017.

18. Vuddanda PR, Chakraborty S and Singh S: Berberine: A potential phytochemical with multispectrum therapeutic activities. Expert Opin Invest Drugs 9: 1297-1307, 2010.

19. Hang Y, Qin X, Ren T and Cao J: Baicalin reduces blood lipids and inflammation in patients with coronary artery disease and rheumatoid arthritis: A randomized, double-blind, placebo-controlled trial. Lipids Health Dis 17: 146, 2018.

20. Ferk F, Kundi M, Brath H, Szekeres T, Al-Serori H, Mišík M, Saiko P, Marculescu R, Wagner KH and Knasmueller S: Gallic acid improves health-associated biochemical parameters and prevents oxidative damage of DNA in type 2 diabetes patients: Results of a placebo-controlled pilot study. Mol Nutr Food Res 62: Jan 18, 2018 (Epub ahead of print). doi: 10.1002/ mnfr.201700482.

21. McCubrey JA, Lertpiriyapong K, Steelman LS, Abrams SL, Yang LV, Murata RM, Rosalen PL, Scalisi A, Neri LM, Cocco L, et al: Effects of resveratrol, curcumin, berberine and other nutraceuticals on aging, cancer development, cancer stem cells and microRNAs. Aging (Albany NY) 9: 1477-1536, 2017.

22. Kang M, Kim S and Ko J: Roles of CD133 in microvesicle formation and oncoprotein trafficking in colon cancer. FASEB J 33: 4248-4260, 2019. 
23. Zhang SS, Han ZP, Jing YY, Tao SF, Li TJ, Wang H, Wang Y, Li R, Yang Y, Zhao X, et al: CD133(+)CXCR4(+) colon cancer cells exhibit metastatic potential and predict poor prognosis of patients. BMC Med 10: 85, 2012.

24. Lu P, Weaver VM and Werb Z: The extracellular matrix: A dynamic niche in cancer progression. J Cell Biol 196: 395-406, 2012.

25. Zucker S and Vacirca J: Role of matrix metalloproteinases (MMPs) in colorectal cancer. Cancer Metastasis Rev 23: 101-117, 2004.

26. De Craene B and Berx G: Regulatory networks defining EMT during cancer initiation and progression. Nat Rev Cancer 13: 97-110, 2013

27. Han YT, Chen XH, Gao H, Ye JL and Wang CB: Physcion inhibits the metastatic potential of human colorectal cancer SW620 cells in vitro by suppressing the transcription factor SOX2. Acta Pharmacol Sin 37: 264-275, 2016.

28. Beyes S, Andrieux G, Schrempp M, Aicher D, Wenzel J, Antón-García P, Boerries $M$ and Hecht A: Genome-wide mapping of DNA-binding sites identifies stemness-related genes as directly repressed targets of SNAIL1 in colorectal cancer cells. Oncogene 38: 6647-6661, 2019.

29. Chen T, You Y, Jiang H and Wang ZZ: Epithelial-mesenchymal transition (EMT): A biological process in the development, stem cell differentiation, and tumorigenesis. J Cell Physiol 232: 3261-3272, 2017

30. Zhao GX, Xu YY, Weng SQ, Zhang S, Chen Y, Shen XZ, Dong L and Chen S: CAPS1 promotes colorectal cancer metastasis via Snail mediated epithelial mesenchymal transformation. Oncogene 38: 4574-4589, 2019.

31. Kahlert C, Lahes S, Radhakrishnan P, Dutta S, Mogler C, Herpel E, Brand K, Steinert G, Schneider M, Mollenhauer M, et al: Overexpression of ZEB2 at the invasion front of colorectal cancer is an independent prognostic marker and regulates tumor invasion in vitro. Clin Cancer Res 17: 7654-7663, 2011.

32. Li MZ, Wang JJ, Yang SB, Li WF, Xiao LB, He YL and Song XM: ZEB2 promotes tumor metastasis and correlates with poor prognosis of human colorectal cancer. Am J Transl Res 9: 2838-2851, 2017

33. Wu K, Wei P, Liu M, Liang X and Su M: To reveal pharmacological targets and molecular mechanisms of curcumol against interstitial cystitis. J Adv Res 20: 43-50, 2019.

34. Cheng F, Kovacs IA and Barabasi AL: Network-based prediction of drug combinations. Nat Commun 10: 1197, 2019.

35. Wang Y, Wang S and Huang M: Structure and enzymatic activities of human serum albumin. Curr Pharm Des 21: 1831-1836, 2015.

36. Bauer $J$ and Capra S: Comparison of a malnutrition screening tool with subjective global assessment in hospitalised patients with cancer-sensitivity and specificity. Asia Pac J Clin Nutr 12: 257-260, 2003

37. McMillan DC, Watson WS, O'Gorman P, Preston T, Scott HR and McArdle CS: Albumin concentrations are primarily determined by the body cell mass and the systemic inflammatory response in cancer patients with weight loss. Nutr Cancer 39: 210-213, 2001.

38. Yamamoto M, Saito H, Uejima C, Tanio A, Tada Y, Matsunaga T, Sakamoto T, Honjo S, Ashida K and Fujiwara Y: Combination of serum albumin and cholinesterase levels as prognostic indicator in patients Ith colorectal cancer. Anticancer Res 39: 1085-1090, 2019.

39. Pal I and Mandal M: PI3K and Akt as molecular targets for cancer therapy: Current clinical outcomes. Acta Pharmacol Sin 33: 1441-1458, 2012

40. Lossi L, Castagna C and Merighi A: Caspase- 3 mediated cell death in the normal development of the mammalian cerebellum. Int J Mol Sci 19: 3999, 2018.

41. Chen H, Yang X, Feng Z, Tang R, Ren F, Wei K and Chen G: Prognostic value of caspase-3 expression in cancers of digestive tract: A meta-analysis and systematic review. Int J Clin Exp Med 8: 10225-10234, 2015.
42. Lieu C and Kopetz S: The SRC family of protein tyrosine kinases: A new and promising target for colorectal cancer therapy. Clin Colorectal Cancer 9: 89-94, 2010.

43. Parkin A, Man J, Timpson P and Pajic M: Targeting the complexity of Src signalling in the tumour microenvironment of pancreatic cancer: From mechanism to therapy. FEBS J 286: 3510-3539, 2019.

44. Kopetz S: Targeting SRC and epidermal growth factor receptor in colorectal cancer: Rationale and progress into the clinic Gastrointest Cancer Res 1 (Suppl 2): S37-S41, 2007.

45. Maa MC, Leu TH, McCarley DJ, Schatzman RC and Parsons SJ: Potentiation of epidermal growth factor receptor-mediated oncogenesis by c-Src: Implications for the etiology of multiple human cancers. Proc Natl Acad Sci USA 92: 6981-6985, 1995.

46. Poole EM, Curtin K, Hsu L, Kulmacz RJ, Duggan DJ, Makar KW, Xiao L, Carlson CS, Slattery ML, Caan BJ, et al: Genetic variability in EGFR, Src and HER2 and risk of colorectal adenoma and cancer. Int J Mol Epidemiol Genet 2: 300-315, 2011

47. Chen J, Elfiky A, Han M, Chen C and Saif MW: The role of Src in colon cancer and its therapeutic implications. Clin Colorectal Cancer 13: 5-13, 2014.

48. Lin L, Cheng K, He Z, Lin Q, Huang Y, Chen C, Xie Z, Chen L and Liang Z: A polysaccharide from hedyotis diffusa interrupts metastatic potential of lung adenocarcinoma A549 cells by inhibiting EMT via EGFR/Akt/ERK signaling pathways. Int J Biol Macromol 129: 706-714, 2019.

49. Geethadevi A, Parashar D, Bishop E, Pradeep S and Chaluvally-Raghavan P: ERBB signaling in CTCs of ovarian cancer and glioblastoma. Genes Cancer 8: 746-751, 2017.

50. Sigismund S, Avanzato D and Lanzetti L: Emerging functions of the EGFR in cancer. Mol Oncol 12: 3-20, 2018

51. Zhang L, Tao X, Fu Q, Ge C, Li R, Li Z, Zhu Y, Tian H, Li Q, Liu M, et al: Curcumin inhibits cell proliferation and migration in NSCLC through a synergistic effect on the TLR4/MyD88 and EGFR pathways. Oncol Rep 42: 1843-1855, 2019.

52. Li C, Cai G, Song D, Gao R, Teng P, Zhou L, Ji Q, Sui H, Cai J, Li Q and Wang Y: Development of EGFR-targeted evodiamine nanoparticles for the treatment of colorectal cancer. Biomater Sci 7: 3627-3639, 2019.

53. Wang N, Pan W, Zhu M, Zhang M, Hao X, Liang G and Feng Y: Fangchinoline induces autophagic cell death via p53/sestrin2/AMPK signalling in human hepatocellular carcinoma cells. Br J Pharmacol 164: 731-742, 2011.

54. Suman S, Kurisetty V, Das TP, Vadodkar A, Ramos G, Lakshmanaswamy R and Damodaran C: Activation of AKT signaling promotes epithelial-mesenchymal transition and tumor growth in colorectal cancer cells. Mol Carcinog 53 (Suppl 1): E151-E160, 2014

55. Shi J, Guo B, Hui Q, Chang P and Tao K: Fangchinoline suppresses growth and metastasis of melanoma cells by inhibiting the phosphorylation of FAK. Oncol Rep 38: 63-70, 2017.

56. Xia J, Chen J, Zhang Z, Song P, Tang W and Kokudo N: A map describing the association between effective components of traditional Chinese medicine and signaling pathways in cancer cells in vitro and in vivo. Drug Discov Ther 8: 139-153, 2014.

57. Bahrami B, Hojjat-Farsang M, Mohammadi H, Anvari E, Ghalamfarsa G, Yousefi M and Jadidi-Niaragh F: Nanoparticles and targeted drug delivery in cancer therapy. Immunol Lett 190: 64-83, 2017.

This work is licensed under a Creative Commons Attribution-NonCommercial-NoDerivatives 4.0 International (CC BY-NC-ND 4.0) License. 